\title{
Traveling for heart transplantation and returning with COVID-19: a logistical, clinical, and pharmacotherapeutic challenge from the Middle East
}

\author{
Bassam Atallah $^{1,2} \cdot$ Iman Hamour ${ }^{3} \cdot$ Saad I. Mallah $^{4} \cdot$ Maria-Fernanda Bonilla $^{5} \cdot$ Feras Bader $^{2,3}$
}

Published online: 31 October 2020

(c) Springer Nature Switzerland AG 2020

\begin{abstract}
Heart transplantation (HT) has become a standard option for patients with end-stage heart failure (HF). However, the scarcity of donor availability remains a major hurdle for receiving this novel therapy, especially in the context of the rapidly spreading severe acute respiratory syndrome coronavirus 2 (SARS-CoV-2; COVID-19) pandemic. We report the case of a patient in the United Arab Emirates (UAE) with advanced HF who was glucose-6-phosphate dehydrogenase deficient and had a history of type 2 diabetes mellitus with diabetic retinopathy and nephropathy, chronic kidney disease stage II, and hyperlipidemia. He was referred for HT abroad and was subsequently caught in the midst of the COVID-19 pandemic in New York, the US state most affected by the crisis at the time. Despite limited experience with favipiravir, we judged it to be the most appropriate agent with this patient's complex history given the lower risk for QT prolongation, no need for renal-dose adjustment, and no reported drug-drug interactions. Given the limited clinical experience with this agent, particularly for our patient, we decided to adopt strategies to mitigate and monitor the potential for QT prolongation. We outline the logistical, clinical, and pharmacological challenges that the poly-morbid patient and our HT program in the Middle-East faced under those novel circumstances.
\end{abstract}

Bassam Atallah

atallab@ccaduae.ae; atallab@clevelandclinicabudhabi.ae

Iman Hamour

Hamouri@ccaduae.ae

Saad I. Mallah

saad.imallh@gmail.com

Maria-Fernanda Bonilla

bonillm@ccaduae.ae

Feras Bader

baderf@ccaduae.ae

1 Department of Pharmacy Services, Cleveland Clinic Abu Dhabi, Al Maryah Island, PO Box 112412, Abu Dhabi, UAE

2 Cleveland Clinic Lerner College of Medicine of Case Western Reserve University, Cleveland, OH, USA

3 Cleveland Clinic Abu Dhabi, Heart and Vascular Institute, Al Maryah Island, PO Box 112412, Abu Dhabi, UAE

4 School of Medicine, Royal College of Surgeons in Ireland, Bahrain, Kingdom of Bahrain

5 Department of Infectious Disease, Cleveland Clinic Abu Dhabi, Al Maryah Island, PO Box 112412, Abu Dhabi, UAE

\section{Introduction}

Heart transplantation (HT) has become a standard option for patients with end-stage heart failure (HF). Survival rates exceed those achieved by medical therapy alone [1]. The scarcity of donor availability remains a major hurdle for receiving this novel therapy [2], and prolonged waiting time on the transplant waiting list is associated with an annual mortality rate of $13-17 \%$ [1-4].

The estimated prevalence of HF in the Middle East Gulf region is around $2 \%$, with a total of 1 million patients, including around 50,000 with advanced HF. It is possible that at least 500 patients may need HT annually in the Middle East Gulf region [5, 6]. To overcome donor scarcity in our newly established HT program-which is the only HT center in the United Arab Emirates (UAE) - we adopted a protocol at Cleveland Clinic Abu Dhabi whereby some patients eligible for HT are referred abroad for surgery. Postoperatively, patients return to the UAE to receive comprehensive post-transplant care and follow-up [7].

We share the case of a patient who was referred for HT abroad and subsequently caught in the midst of the severe acute respiratory syndrome coronavirus 2 (SARS-CoV-2; 
COVID-19) pandemic in New York City, NY, the US state most affected by the crisis at the time. We outline the logistical, clinical, and pharmacological challenges that the patient and our program faced under those circumstances.

\section{Case report}

A 41-year-old man presented to our institution with advanced HF stage D, New York Heart Association (NYHA) functional class IV. At 1 month before his presentation, he sustained an extensive anterior wall ST-elevation myocardial infarction. Cardiac catheterization at the time revealed acute proximal left anterior descending artery occlusion, for which he underwent coronary angioplasty with stent placement. His initial acute presentation was complicated by cardiogenic shock and acute kidney and liver injury requiring inotropic support. At 5 days following his acute presentation, he developed acute hemolytic anemia secondary to aspirin therapy in the setting of inherited glucose-6-phosphate dehydrogenase (G6PD) deficiency. Aspirin was discontinued, and he remained on ticagrelor. Within 10 days, he was discharged home but continued to have severe left ventricular impairment, with a left ventricular ejection fraction (LVEF) of $25-30 \%$ by transthoracic echocardiography. He had a past medical history of type 2 diabetes mellitus with diabetic retinopathy, diabetic nephropathy, chronic kidney disease (CKD) stage II, and hyperlipidemia.

Following discharge, guideline-directed medical therapy was started for HF. This included low doses of a $\beta$-blocker and an angiotensin-receptor blocker, along with a target-dose mineralocorticoid receptor antagonist, in line with the 2017 American College of Cardiology/American Heart Association/Heart Failure Society of America guideline for the management of HF [8]. Unfortunately, he remained at NYHA functional class IV and underwent advanced HF therapy assessment. He was accepted on the HT waiting list and referred to New York City for cardiac transplantation. On arrival, he slowly developed cardiac decompensation with poor organ perfusion, requiring inotropic support followed by temporary mechanical support in the form of intra-aortic balloon pump (IABP) for further stabilization; he was then placed on the urgent HT waiting list. He remained on the waiting list for a full month, during which his HF stabilized and the IABP was removed. Although he did not undergo an HT, his inotropic requirements decreased and end organ function improved.

Around this time, the COVID-19 pandemic continued to escalate, severely overwhelming hospitals and healthcare systems in general. As a result, all HT operations in New York were halted, and the patient was advised to travel back to the UAE, where the pandemic situation was much more controlled. His transfer was accepted to our institution.
At $48 \mathrm{~h}$ before his transfer, he developed a fever of $104 \mathrm{~F}^{\circ}$ and a mild sore throat, which subsided with no recurrence. A SARS-CoV-2 reverse transcription polymerase chain reaction (RT-PCR) performed in New York was negative so he was cleared to travel. On arrival to the UAE, he was afebrile, and the repeat SARS-CoV-2 RT-PCR test was negative. He denied shortness of breath or cough; a transthoracic echocardiogram confirmed severe left ventricular dysfunction with an LVEF of $33 \%$. His chest X-ray showed a small basal shadow consistent with consolidation (Fig. 1).

A third nasopharyngeal and oropharyngeal swab for SARS-CoV-2 RT-PCR returned a positive result. We kept the patient in an extended airborne precaution environment from the time of his admission. His electrocardiogram (ECG) showed sinus rhythm with no conduction defects, a corrected QT (QTc) interval of $435 \mathrm{~ms}$, and features consistent with his previous extensive myocardial infarction (Fig. 2).

His laboratory results revealed normocytic anemia, low white blood cell count with associated lymphopenia, elevated inflammatory and cardiac markers, including $\mathrm{N}$-terminal pro-brain natriuretic peptide, troponin, ferritin, procalcitonin, and D dimer. He had an effective glomerular filtration rate (eGFR) of $24 \mathrm{~mL} / \mathrm{min}$ and low serum sodium levels (Table 1).

In view of his critical cardiac condition, we started therapy with favipiravir. Factors considered in the choice of antiviral therapy included his increased risk of ventricular arrhythmias, history of hemolysis secondary to G6PD deficiency, and therapy with ticagrelor, all of which excluded hydroxychloroquine, lopinavir/ritonavir, and azithromycin. He received 5 days of oral favipiravir therapy $(1600 \mathrm{mg}$ every $12 \mathrm{~h}$ on day 1 as a loading dose, followed by $600 \mathrm{mg}$ every $8 \mathrm{~h}$ for 10 days), during which repeated nasopharyngeal swabs for SARS-CoV-2 RT-PCR were negative on two

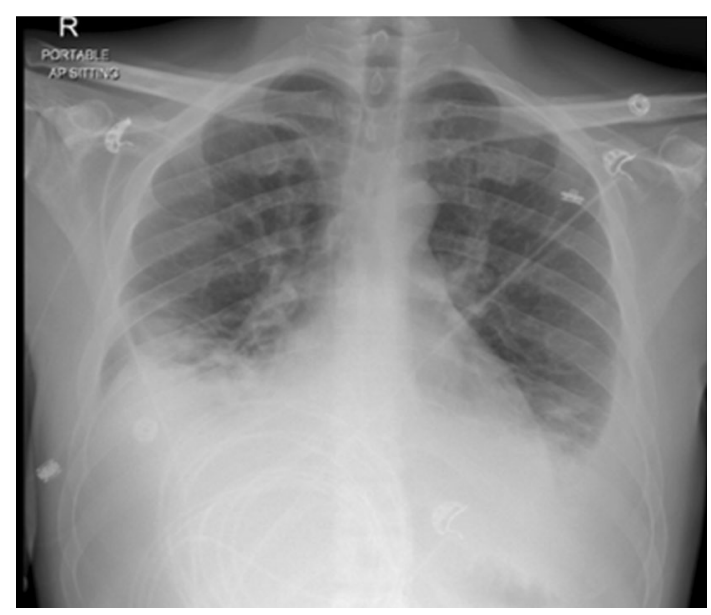

Fig. 1 Postero-anterior chest radiograph 


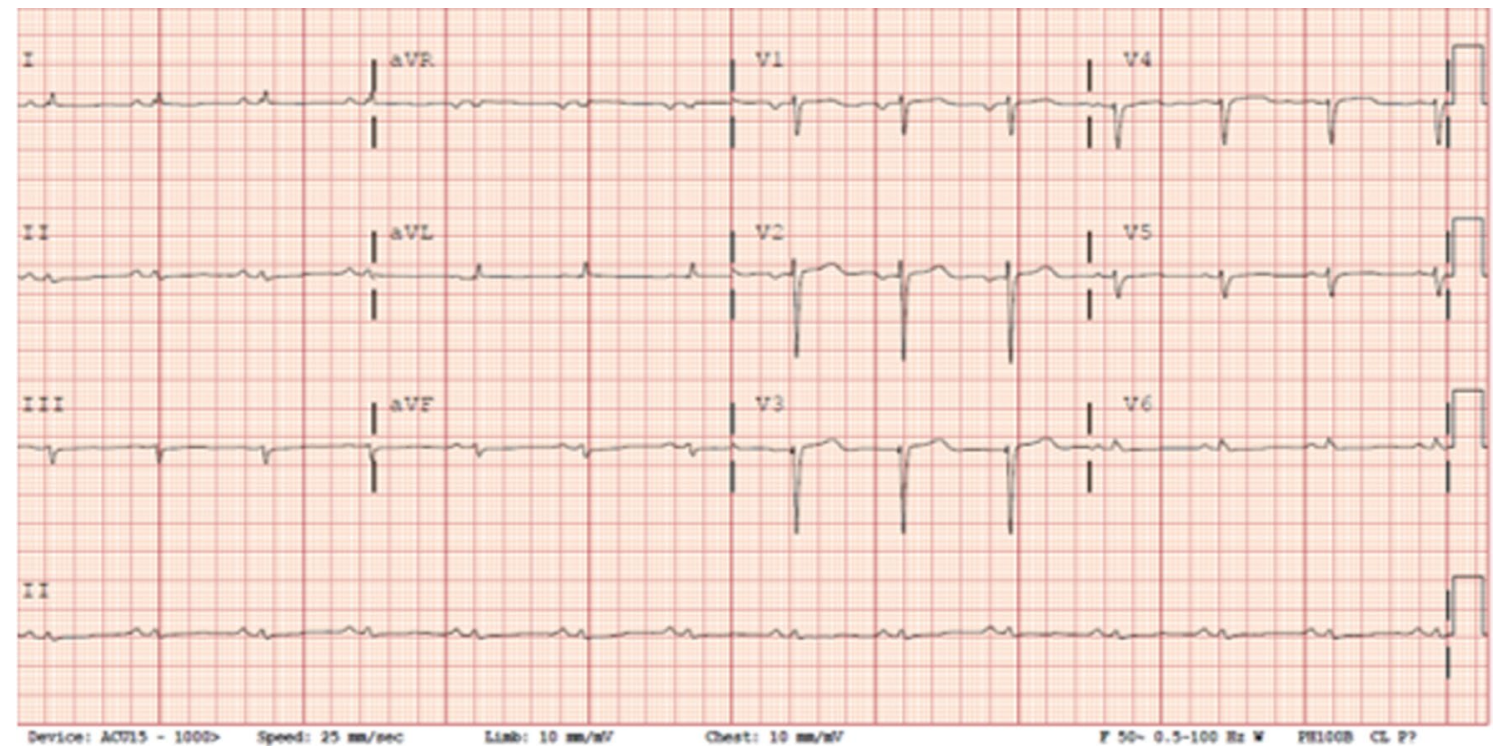

Fig. 2 Baseline electrocardiogram

Table 1 Laboratory parameters on admission upon returning from New York, USA

\begin{tabular}{|lll}
\hline Laboratory test (unit) & Patient value & Reference range \\
\hline NT-proBNP $(\mathrm{ng} / \mathrm{L})$ & 3707 & $<85.8$ \\
Troponin $\mathrm{T}(\mu \mathrm{g} / \mathrm{L})$ & 0.105 & $<0.06$ \\
\hline High-sensitivity troponin $(\mathrm{ng} / \mathrm{L})$ & 105 & $<15$ \\
\hline Procalcitonin $(\mu \mathrm{g} / \mathrm{L})$ & 0.10 & $<0.05$ \\
\hline Ferritin $(\mu \mathrm{g} / \mathrm{L})$ & 501 & $36-480$ \\
\hline C-reactive protein $(\mathrm{mg} / \mathrm{L})$ & 4.8 & $<5.00$ \\
\hline D dimer $(\mu \mathrm{g} / \mathrm{mL}$ FEU) & 0.89 & $<0.50$ \\
\hline Lipoprotein $(\mathrm{a})(\mathrm{nmol} / \mathrm{L})$ & $<7.0$ & $<75$ \\
\hline Sodium $(\mathrm{mmol} / \mathrm{L})$ & 133 & $136-145$ \\
\hline Serum creatinine $(\mu \mathrm{mol} / \mathrm{L})$ & 267 & $59-104$ \\
\hline eGFR $\left(\mathrm{mL} / \mathrm{min} / 1.73 \mathrm{~m}{ }^{2}\right)$ & 24 & $>60$ \\
Hematocrit $(\mathrm{SI} \mathrm{units)}$ & 0.30 & $0.39-0.49$ \\
\hline Hemoglobin $(\mathrm{g} / \mathrm{L})$ & 92 & $132-173$ \\
\hline Platelets $\left(\times 10^{9} / \mathrm{L}\right)$ & 256 & $140-400$ \\
\hline WBC $\left(\times 10^{9} / \mathrm{L}\right)$ & 3.45 & $4.5-11.0$ \\
\hline Basophils $\left(\times 10^{9} / \mathrm{L}\right)$ & 0.04 & $0.00-0.15$ \\
\hline Eosinophils $\left(\times 10^{9} / \mathrm{L}\right)$ & 0.16 & $0.00-0.70$ \\
\hline Lymphocytes $\left(\times 10^{9} / \mathrm{L}\right)$ & 0.62 & $1.50-4.00$ \\
\hline Neutrophils $\left(\times 10^{9} / \mathrm{L}\right)$ & 2.20 & $1.80-7.70$ \\
\hline
\end{tabular}

eGFR effective glomerular filtration rate, $F E U$ fibrinogen equivalent units, $N T$-proBNP N-terminal pro-brain natriuretic peptide; $W B C$ white blood cells

occasions. During this episode, we carefully monitored his QTc interval. He remained in compensated HF with no episodes of any ventricular arrhythmias, and we eventually weaned off and discontinued his inotropic therapy.
However, his lymphocyte count dropped during the favipiravir course; this was attributed to this medication, which was thus stopped after a 5-day course. We stabilized him sufficiently to allow discharge to home to complete the 2 weeks of required self-quarantine. The patient stayed at the UAE hospital for a total of 9 days.

On discharge to homecare, the patient's condition was stable with no signs of distress and was at NYHA functional class II. Nasopharyngeal and sputum tests for COVID-19 were negative. His functional capacity has improved since discharge and he has started regular physical therapy. $\mathrm{He}$ continues to receive follow-up in our clinic and is planned for another admission for full HT workup and reassessment of listing for $\mathrm{HT}$ in the UAE.

\section{Discussion}

Our institutional protocol for the management of COVID-19 pneumonia at the time included the oral combination antiviral lopinavir/ritonavir $400 \mathrm{mg} / 100 \mathrm{mg}$ twice daily for 10 days in addition to oral hydroxychloroquine $400 \mathrm{mg}$ twice daily on day 1 , followed by $400 \mathrm{mg}$ daily for a total of 5-10 days (Table 2). This protocol was based on the very limited literature available at the time. However, the first-line agents in this protocol were clearly not an option for our patient. Considerations that played a role in the treatment decision for this patient included his G6PD status, the risk for QT prolongation, and drug-drug interactions. This resulted in using a second-line therapy of oral favipiravir $1600 \mathrm{mg}$ every $12 \mathrm{~h}$ on day 1 as a loading dose, followed by $600 \mathrm{mg}$ every $8 \mathrm{~h}$ for 10 days $[9,10]$. Our current protocol as of October 
Table 2 Our institutional protocol for the treatment of patients with COVID-19 with pneumonia at the time of case admission ${ }^{\mathrm{a}}$

\begin{tabular}{|c|c|}
\hline \multicolumn{2}{|c|}{$\begin{array}{l}\text { Lopinavir/ritonavir } 400 \mathrm{mg} / 100 \mathrm{mg} \text { ( } 21 \text { tablets) twice daily } \times \\
10-14 \text { days }\end{array}$} \\
\hline \multicolumn{2}{|l|}{ PLUS } \\
\hline \multirow[t]{3}{*}{ First line } & Hydroxychloroquine \\
\hline & Day 1: $400 \mathrm{mg}$ every $12 \mathrm{~h}$ \\
\hline & Then $400 \mathrm{mg} /$ day $\times 9-14$ days \\
\hline \multicolumn{2}{|l|}{ OR } \\
\hline \multirow{3}{*}{$\begin{array}{l}\text { Second line: patients intolerant } \\
\text { to hydroxychloroquine (i.e., } \\
\text { risk of QTc prolongation) }\end{array}$} & Favipiravir \\
\hline & $\begin{array}{l}\text { Day 1: } 1600 \mathrm{mg} \text { ( } 8 \text { tablets) every } \\
12 \mathrm{~h} \text { (loading dose) }\end{array}$ \\
\hline & $\begin{array}{l}\text { Then } 600 \mathrm{mg} \text { ( } 3 \text { tablets }) \text { every } \\
8 \mathrm{~h} \times 9-14 \text { days }\end{array}$ \\
\hline
\end{tabular}

COVID-19 coronavirus 2019 (severe acute respiratory syndrome coronavirus 2 [SARS-CoV-2]), QTc corrected QT interval

${ }^{a}$ Our current protocol recommends favipiravir as first-line therapy for the treatment of mildly symptomatic COVID-19 (mild pneumonia, no cytokine storm, and patient not on supplemental oxygen). Hydroxychloroquine and lopinavir/ritonavir are no longer recommended in our protocol; instead, dexamethasone and remdesivir are used in the treatment of severe COVID-19 pneumonia in patients requiring supplemental oxygen

2020 recommends the use of favipiravir in mild COVID19 and a combination of dexamethasone and remdesivir in severe COVID-19. Hydroxychloroquine and lopinavir/ritonavir are no longer recommended.

While primaquine has been associated with hemolytic anemia in patients with G6PD, other antimalarial drugs, including chloroquine and hydroxychloroquine, are also on the list of medications that can cause this complication [11]. G6PD deficiency has been linked to not only hemolytic anemia but also increased susceptibility to viral infections, including coronaviruses. This impaired immune response is likely because of an abnormal nuclear factor (NF)- $\mathrm{KB}$ signaling and antiviral response mediated by HSCARG protein, a nicotinamide adenine dinucleotide phosphate sensor and negative regulator of $\mathrm{NF}-\mathrm{\kappa B}$, affecting antiviral response [12].

The risk of QT prolongation and torsades de pointes was also significant in this patient and complicated the choice of COVID-19 therapy. His advanced HF status, low cardiac output, and recent dependence on inotropes put him at high risk for sudden cardiac death. To complicate the picture further, our patient was receiving amiodarone when he transferred to us, which was continued until the day of favipiravir initiation. Ventricular arrhythmia is particularly of concern in patients with structural heart disease who are receiving one or more QT-prolonging medications. If we calculated the Tisdale score (a validated risk score to predict QT prolongation in hospitalized patients [13]) for this
Table 3 Tisdale score to predict QT prolongation in hospitalized patients

\begin{tabular}{lc} 
Risk factors $^{\mathrm{a}}$ & Points \\
\hline Age $\geq 68$ years & 1 \\
Female sex & 1 \\
Loop diuretic $^{\mathrm{a}}$ & $\mathbf{1}$ \\
Serum K$^{+} \leq 3.5 \mathrm{mEq} / \mathrm{L}$ & 2 \\
${\text { Admission QTc } \geq \mathbf{4 5 0} \mathbf{~ m s}^{\mathrm{a}}}^{\text {Acute myocardial infarction }}$ & $\mathbf{2}$ \\
$\geq 2$ QTc-prolonging drugs & 2 \\
\hline Sepsis & 3 \\
Heart failure $^{\mathrm{a}}$ & 3 \\
One QTc-prolonging drug $^{\mathrm{a}}$ & $\mathbf{3}$ \\
\hline Maximum total risk score & $\mathbf{3}$ \\
& 21
\end{tabular}

Adapted from Table 5 in Tisdale et al. [11]

Total Tisdale score $\leq 6$ predicts low risk, $7-10$ medium risk, and $\geq 11$ high risk of drug-associated QT prolongation

$Q T c$ corrected QT interval

${ }^{a}$ Bolded risk factors indicate risk factors present in our patient, who had a total score of 9

patient, a score of at least 9 would have placed him in the medium- to high-risk range for QT prolongation (Table 3). Factors influencing this score in our patient, in addition to his known increased risk due to structural heart disease, include being on a loop diuretic and thus more prone to electrolyte abnormalities. An admission QTc over $450 \mathrm{~ms}$, $\mathrm{HF}$, and amiodarone use all result in a relatively high Tisdale score (Table 3).

Additional considerations that further complicated the choice of therapy in our patient were the drug-drug interactions present at the time of the decision. Having undergone recent percutaneous coronary intervention and stenting, our patient was still receiving dual antiplatelet therapy with aspirin and ticagrelor, amiodarone to prevent arrhythmias, and tolvaptan. We discontinued amiodarone on the day of COVID-19 therapy initiation, as it was judged unnecessary and could be replaced with a $\beta$-blocker; however, as the effect of amiodarone persists for weeks because of its long half-life, it was still considered an interacting agent. The combination antiviral lopinavir/ritonavir would have interacted with ticagrelor and increased the risk of bleeding [14]. This is in addition to the interaction with amiodarone and statin, with tolvaptan being a strong CYP3A4 inhibitor and having the potential to prolong the QT (according to the CredibleMeds list) [15]. Similarly, hydroxychloroquine was contraindicated because of the G6PD deficiency and potential for QT prolongation, particularly in combination with amiodarone. Thus, even though the previously mentioned 
medications are the first-line therapy for COVID-19 per our institutional protocol, we avoided them in this case.

Despite limited experience with favipiravir, we judged it to be the most appropriate agent in this scenario, given the lower risk for QT prolongation, no need for renal-dose adjustments, and no reported drug-drug interactions. Favipiravir was reported as not having caused QT prolongation after single oral doses of $1200 \mathrm{mg}$ and $2400 \mathrm{mg}$ [16]. However, in one case report in the treatment of Ebola virus, favipiravir prolonged the QT at higher doses [17]. Given the limited clinical experience with this agent, particularly for our patient, we decided to use strategies to mitigate and monitor the potential for QT prolongation. First, we discontinued all other QT-prolonging agents, including amiodarone and the as-needed ondansetron. The patient was placed on telemetry, with baseline ECG obtained before initiation, $2 \mathrm{~h}$ after the first dose, and $2 \mathrm{~h}$ after each morning dose. Baseline and daily laboratory tests were conducted to monitor electrolyte abnormalities, with the goal of keeping potassium levels $>4$ $\mathrm{mEq} / \mathrm{L}$ and magnesium levels $>2 \mathrm{mg} / \mathrm{dL}(0.8 \mathrm{mmol} / \mathrm{L})$.

Our protocol was based on the limited literature for this agent available at the time. Unpublished internal data from our center suggest that favipiravir may help in mild/moderate disease, but-as with other antivirals-its efficacy in later stages of disease (e.g., when mechanical ventilation is required) is lacking. We thus judged favipiravir to be an option for mild pneumonia and for patients with high risk for disease progression, particularly in the setting of contraindications to and interactions with other agents. More data and randomized clinical trials are needed to judge the efficacy of our approach and the best treatments for complex scenarios.

\section{Take home messages}

- Logistic and clinical difficulties are encountered by patients with advanced HF who need HT in areas where it may not be readily available, particularly in a pandemic situation.

- When selecting antiviral treatment for COVID-19 in patients with advanced HF, carefully consider potential cardiovascular adverse events and drug-drug interactions.

- G6PD deficiency and commonly used medications in patients with advanced HF can present a particular challenge for COVID-19 antiviral therapy.

- Despite limited clinical experience with the antiviral agent favipiravir, its use early in mild/moderate COVID19 may be considered in patients with advanced HF with careful monitoring of their QT interval.

\section{Declarations}

Funding No funding was obtained for this manuscript.

Conflicts of interest Bassam Atallah, Iman Hamour, Saad I. Mallah, Maria-Fernanda Bonilla, and Feras Bader have no conflicts of interest that are directly relevant to the content of this article.

Ethics approval Our institution does not require ethics approval for case reports and thus this requirement was waived.

Consent to participate Not applicable

Consent for publication Verbal consent acquired.

Availability of data and material Available upon request.

Code availability Not applicable.

Author contributions All authors contributed to the drafting and revision of the manuscript and approve the final version.

\section{References}

1. Alraies MC, Eckman P. Adult heart transplant: indications and outcomes. Thorac Dis. 2014;6(8):1120-8.

2. Chambers DC, Cherikh WS, Harhay MO, et al. The International Thoracic Organ Transplant Registry of the International Society for Heart and Lung Transplantation: thirty-sixth adult lung and heart-lung transplantation report-2019; focus theme: donor and recipient size match. J Heart Lung Transplant. 2019;38(10):1042-55.

3. Hamour I, Khaghani A, Kanagala P, et al. Current outcome of heart transplantation: a 10-year single center perspective and review. QJM. 2011;104(4):335-43.

4. Scientific Registry of Transplant Recipients. 2009 annual report of the U.S. Organ Procurement and Transplantation Network and the Scientific Registry of Transplant Recipients: transplant data 1999-2008. Rockville (MD): U.S. Department of Health \& Human Services.

5. Bader F, Atallah B, Rizk J. Heart transplantation in the Middle East Gulf region. Transplantation. 2018;102(7):1023-6.

6. Al Shamiri MQ. Heart failure in the Middle East. Curr Cardiol Rev. 2013;9:174-8.

7. Hamour IM, Ferrer R, AbuBakr S, et al. Beyond borders: our Middle Eastern experience of international collaboration to run a successful heart transplantation program. J Card Fail. 2018;24(8):S127.

8. Yancy CW, Jessup M, Bozkurt B, et al. 2017 ACC/AHA/HFSA focused update of the 2013 ACCF/AHA guideline for the management of heart failure: a report of the American College of Cardiology/American Heart Association Task Force on Clinical Practice Guidelines and the Heart Failure Society of America. J Am Coll Cardiol. 2017;70(6):776-803.

9. Cai Q, Yang M, Liu D, et al. Experimental treatment with favipiravir for COVID-19: an open-label control study. Engineering (Beijing). 2020. https://doi.org/10.1016/j.eng.2020.03.007.

10. Chen C, Huang J, Cheng Z, et al. Favipiravir versus arbidol for COVID-19: a randomized clinical trial. MedRxiv. 2020. https:// doi.org/10.1101/2020.03.17.20037432. 
11. Luzzatto L, Seneca E. G6PD deficiency: a classic example of pharmacogenetics with on-going clinical implications. Br J Haematol. 2014;164(4):469-80.

12. Wu YH, Chiu DT, Lin HR, et al. Glucose-6-phosphate dehydrogenase enhances antiviral response through downregulation of NADPH sensor HSCARG and upregulation of NF- $\mathrm{KB}$ signaling. Viruses. 2015;7(12):6689-706.

13. Tisdale JE, Jaynes HA, Kingery JR, et al. Development and validation of a risk score to predict QT interval prolongation in hospitalized patients. Circ Cardiovasc Qual Outcomes. 2013;6(4):479-87.
14. Egan G, Hughes CA, Ackman ML. Drug interactions between antiplatelet or novel oral anticoagulant medications and antiretroviral medications. Ann Pharmacother. 2014;48(6):734-40.

15. Woosley RL, Heise CW, Gallo T, et al. QTDrugs lists. www.Credi bleMeds.org. Accessed 11 Oct 2020

16. Kumagai Y, Murakawa Y, Hasunuma T, et al. Lack of effect of favipiravir, a novel antiviral agent, on QT interval in healthy Japanese adults. Int J Clin Pharmacol Ther. 2015;53(10):866-74.

17. Chinello P, Petrosillo N, Pittalis S, et al. QTc interval prolongation during favipiravir therapy in an Ebolavirus-infected patient. PLoS Negl Trop Dis. 2017;11(12):e0006034. 